\title{
Estenosis mitral con calcificación severa del anillo mitral. Reemplazo mitral con prótesis OnX Conform en posición supraanular
}

\author{
Romina Novillo ${ }^{1}$, Matías Macedo ${ }^{1}$, Luigi Gabrielli $^{1,2}$, Bernardita Garayar $^{1}$, Julián Vega $^{1}$, Samuel Córdova ${ }^{1}$, Ricardo Zalaquett $^{2,3}$. \\ 1. Facultad de Medicina. División de Enfermedades Cardiovasculares. Pontificia Universidad Católica de Chile. \\ 2. Advanced Center for Chronic Diseases (ACCDiS). \\ 3. Facultad de Medicina. Sección Cirugía Cardíaca. División de Cirugía. Pontificia Universidad Católica de Chile. \\ Financiamiento: Ninguno. \\ Conflictos de interés: ninguno
}

\section{Mitral stenosis with severe annular calcification. Mitral valve replacement with an OnX Conform prosthesis in a supra annular position.}

A 68-year-old man previously subjected to radiotherapy had a prior aortic valve replacement due de radiation induced calcification of the aortic valve. Presently the patient developed severe calcification of the mitral valve ring leading to critical mitral valve stenosis. A supra annular implantation of an On X Conform valve was successfu- lly achieved. The clinical course was uneventful, and the echocardiographic evaluation demonstrated a normal function of the valve. Different alternatives for the surgical management of this complication are discussed.

Keywords: Mitral Stenosis, Mitral Annular Calcification, Mitral Valve Replacement. 


\section{Caso Clínico:}

Se presenta el caso clínico de un paciente hombre de 68 años, con antecedentes de tratamiento con radioterapia en 1969 por cáncer pulmonar y mediastínico y un reemplazo valvular aórtico con prótesis mecánica por estenosis aórtica actínica, en 2003.

Posteriormente, presenta insuficiencia cardíaca progresiva, que se intensifica en el último tiempo, como consecuencia de haber desarrollado una estenosis mitral severa, por lo que es derivado por su médico tratante a nuestra institución para un eventual reemplazo mitral. En el estudio ecocardiográfico se confirmó una estenosis mitral severa, producto de una calcificación muy extensa de los velos y anillo mitral (Figura 1); además, se demostró una insuficiencia tricuspídea significativa e hipertensión pulmonar severa. La prótesis mecánica en posición aórtica se encontraba normo funcionante. El estudio angiográfico para la evaluación de la anato- mía coronaria hizo visualmente muy evidente la severidad de la calcificación del aparato valvular mitral y en especial de su anillo (Figura 2).

Luego de realizar estudios complementarios se discutió el caso en "heart team" y se decidió conducta quirúrgica, la que fue aceptada por el paciente, en pleno conocimiento de riesgos y beneficios.

Durante la cirugía se evidenció el corazón muy firmemente adherido al saco pericárdico, el que había sido cerrado en la operación previa, y que, además, presentaba cambios inflamatorios de tipo actínico con zonas de engrosamiento importante. Se abordó la aurícula izquierda por un acceso trans septal y superior combinado. Expuesta satisfactoriamente la válvula mitral, se procedió a resecar el velo mitral posterior y parte del velo anterior severamente calcificados y luego a descalcificar parcialmente el anillo mitral posterior, de manera de reducir al máximo el riesgo de disyunción

Figura 1. Ecocardiograma transesofágico tridimensional. Asterisco muestra grandes "mamelones" de calcio en el anillo mitral posterior en la imagen tridimensional.

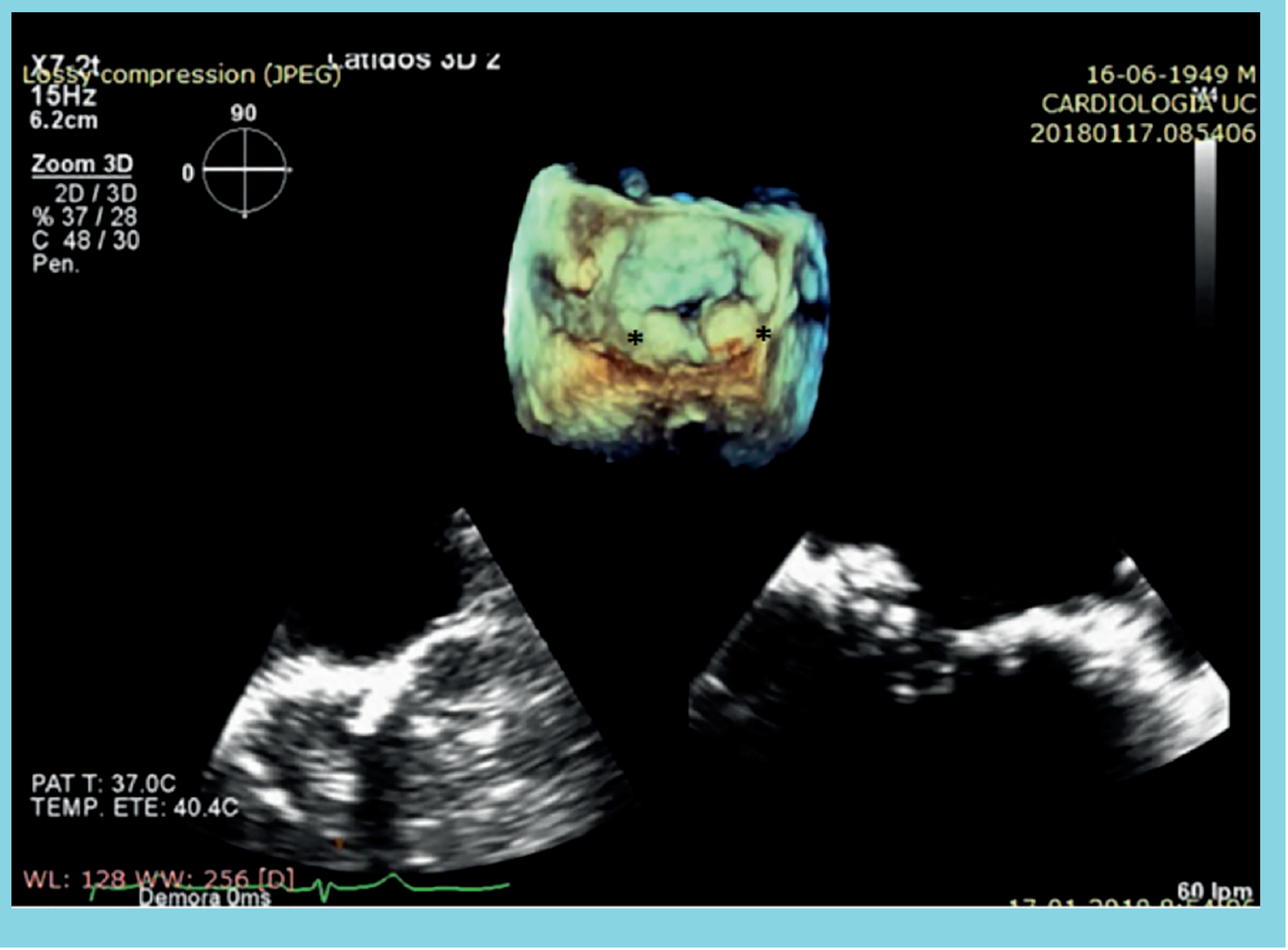


Figura 2. Fluoroscopía que muestra anillo protésico aórtico en situación superior respecto al anillo mitral, el que se observa, a su vez, en extremo calcificado en toda sus circunferencia (asterisco).

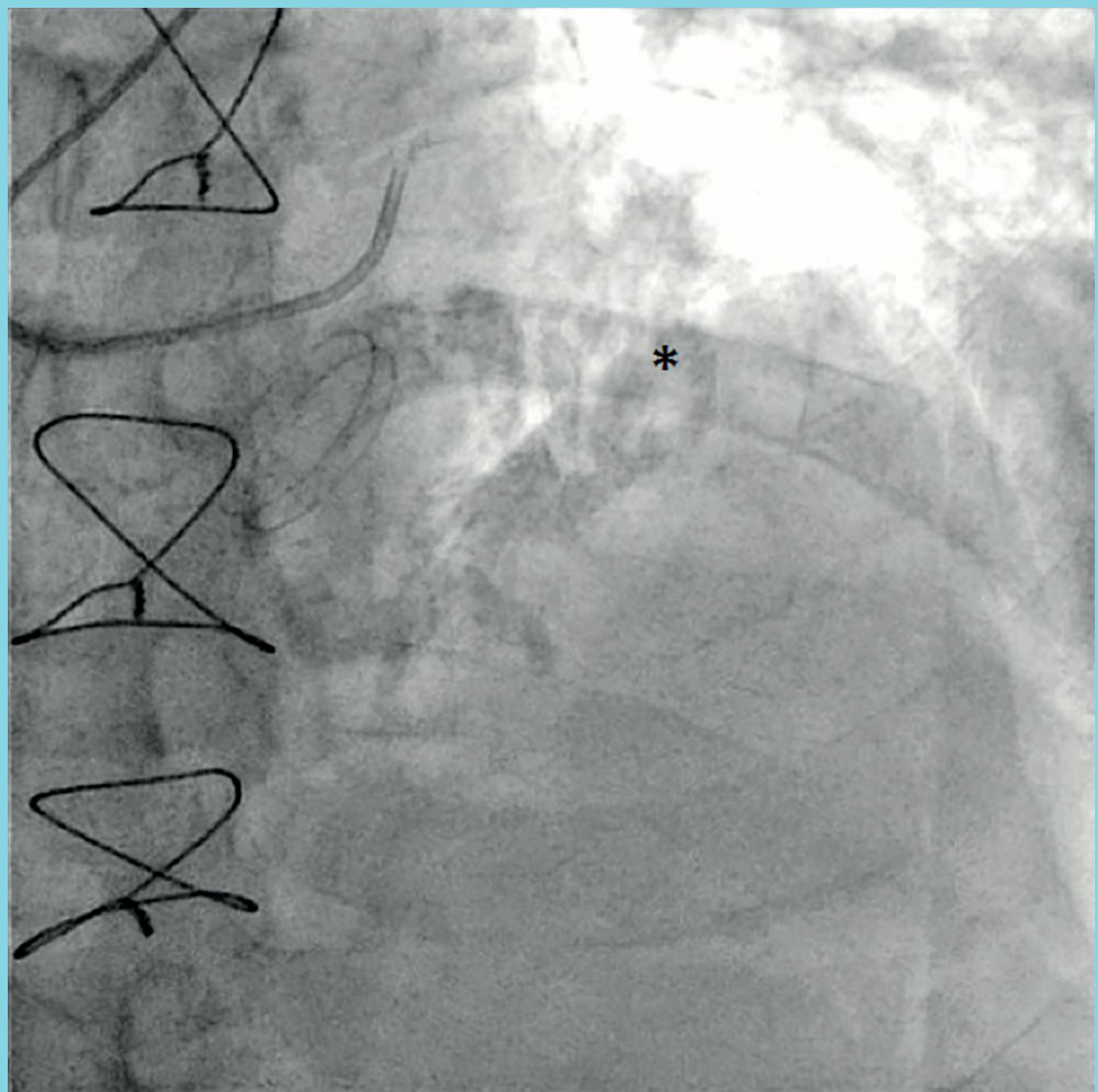

auriculo-ventricular. A continuación, se procedió a efectuar un reemplazo valvular mitral con una prótesis OnX Conform, la que se suturó a la pared auricular izquierda yuxta anular, de manera de asentar la prótesis en posición completamente supra anular. A continuación se efectuó una anuloplastía tricuspídea con un anillo protésico Physio Tricuspídeo de Carpentier-Edwards.

La ecocardiografía transesofágica, tanto intraoperatoria post circulación extracorpórea como al año de la cirugía, evidenció una prótesis mitral en situación supra anular normo funcionante, con gradientes adecuados (Figura 3).

\section{Discusión}

La radioterapia mediastínica se asocia frecuentemente con anomalías valvulares significativas desarrolladas en el transcurso del tiempo (7\%-39\% a 10 años y $12 \%$ $60 \%$ a 20 años), siendo las válvulas del lado izquierdo las más afectadas ${ }^{1}$. Esto, generalmente, se manifiesta en un progresivo engrosamiento y calcificación de la válvula comprometida, lo que resulta, a su vez, en res- 
Figura 3. Ecocardiograma transesofágico intraoperatorio. Se observa prótesis mecánica mitral en situación supraanular.

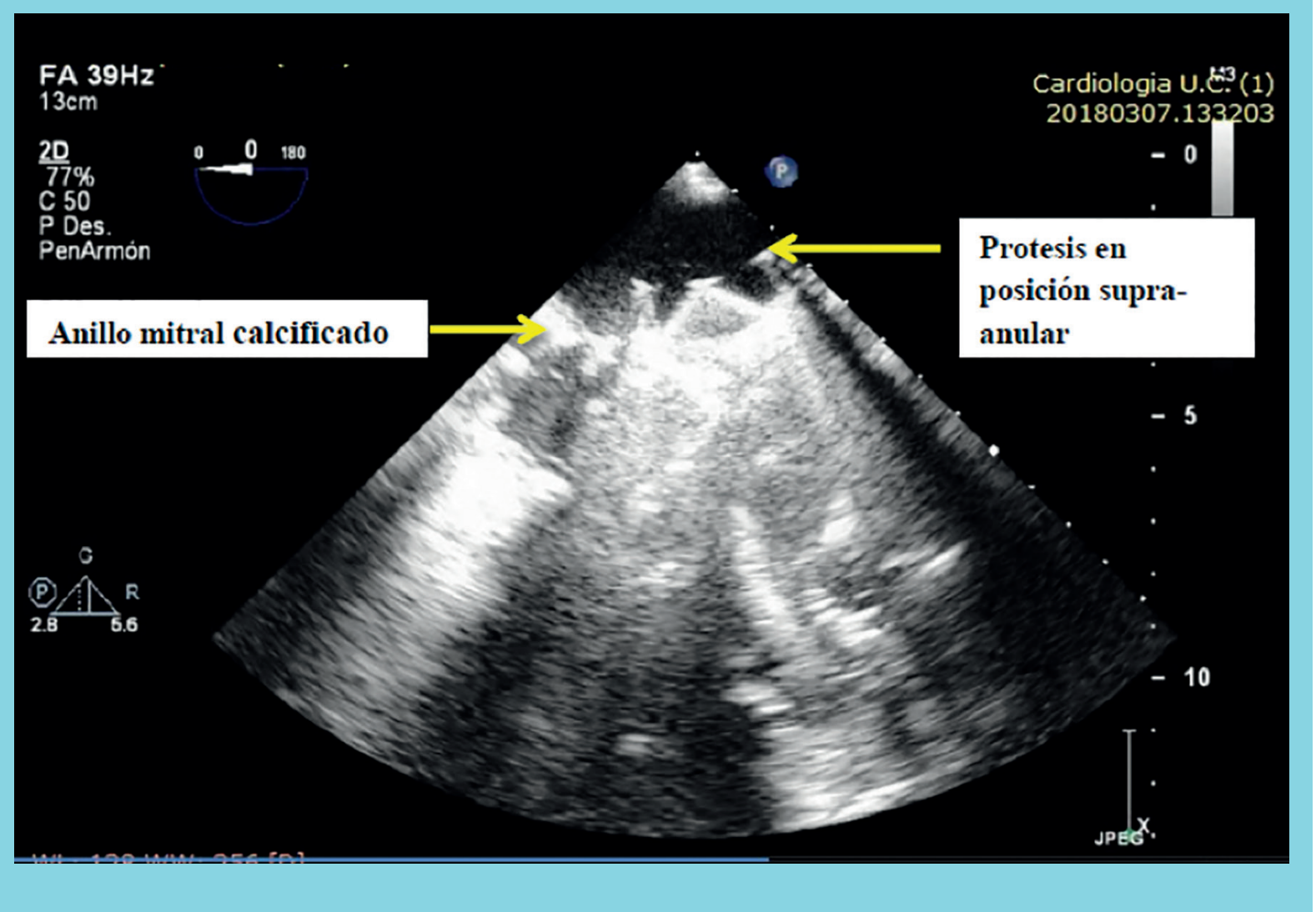

tricción, tanto al cierre como principalmente a la apertura valvular, lo que origina una estenosis valvular, acompañada de cierto grado de regurgitación valvular. El engrosamiento o calcificación de la fascia mitro-aórtica es un signo característico en pacientes que desarrollan cardiopatía actínica y la extensión de ella está asociada con una mortalidad significativa ${ }^{2}$. Existe un escenario clínico frecuente, consistente en que la valvulopatía aórtica actínica es severa y la valvulopatía mitral es moderada, a menudo con calcificación del anillo mitral posterior. En este escenario, algunos estudios recomiendan reemplazar ambas válvulas en una primera intervención, incluso si la enfermedad de una es sólo leve a moderada, dada la tendencia a la calcificación progresiva de la válvula aórtica, fascia mitro-aórtica y anillo valvular mitral $^{3}$, como sucedió en este paciente.

En cuanto a la cirugía valvular en los pacientes con enfermedad cardíaca asociada a radiación, hay dos hallazgos que son de suma importancia, debido a que aumentan el riesgo de complicaciones muy significati- vamente; el primero es, como ya señalamos, la calcificación tisular que se extiende desde el anillo aórtico a través de la fascia mitro-aórtica y que involucra el velo anterior y el anillo de la válvula mitral; y el segundo, es el pequeño tamaño del anillo aórtico y del anillo mitral, debido al proceso inflamatorio actínico, crónico y sostenido que lleva a la retracción de los anillos valvulares ${ }^{4}$.

Un anillo mitral severamente calcificado, como el que presentaba nuestro paciente, conlleva un desafío quirúrgico mayor durante el reemplazo de la válvula mitral. Para asegurar la fijación adecuada del anillo de sutura de la prótesis y para evitar la regurgitación peri protésica, el anillo mitral a menudo debe desbridarse para una fijación segura. Sin embargo, el desbridamiento extenso aumenta significativamente el riesgo de disyunción auriculoventricular en la mayoría de los casos, lo que constituye una complicación operatoria catastrófica, prácticamente insalvable.

Se han descrito numerosas técnicas quirúrgicas para efectuar un reemplazo mitral en presencia de un anillo 
mitral extensamente calcificado. Ello revela la magnitud del desafío quirúrgico y la elevada mortalidad. No existe actualmente una real solución a este problema. Algunos cirujanos han usado refuerzo con parche de pericardio del anillo mitral después del desbridamiento y antes de la colocación de la prótesis. Otra técnica es colocar las suturas desde el lado auricular, debajo de las calcificaciones anulares y a través del borde libre de los velos mitrales y luego a través del anillo de sutura de la prótesis. Sin embargo, esta técnica aumenta el riesgo de lesión de la arteria circunfleja. Otra alter- nativa es el uso del velo anterior, si este no está calcificado, para sostener el anillo posterior después del desbridamiento ${ }^{5}$. El consenso entre la mayoría de los cirujanos es que, si es posible no operar a un paciente con una calcificación extensa de su anillo mitral, esta es la mejor opción.

En nuestro paciente, muy sintomático y refractario al tratamiento médico, no hubo otra opción que el reemplazo mitral, como se describió previamente. Este se efectuó con una prótesis mitral OnX Conform (Figura 4). Esta es una prótesis cardíaca mecánica que cons-

Figura 4. Prótesis mitral OnX Conform. Se observa verdadero "cilindro" de grafito dentro del cual se mueven las valvas protésicas y el extenso cojinete de sutura de PTFE (OnX life technologies Inc. USA).

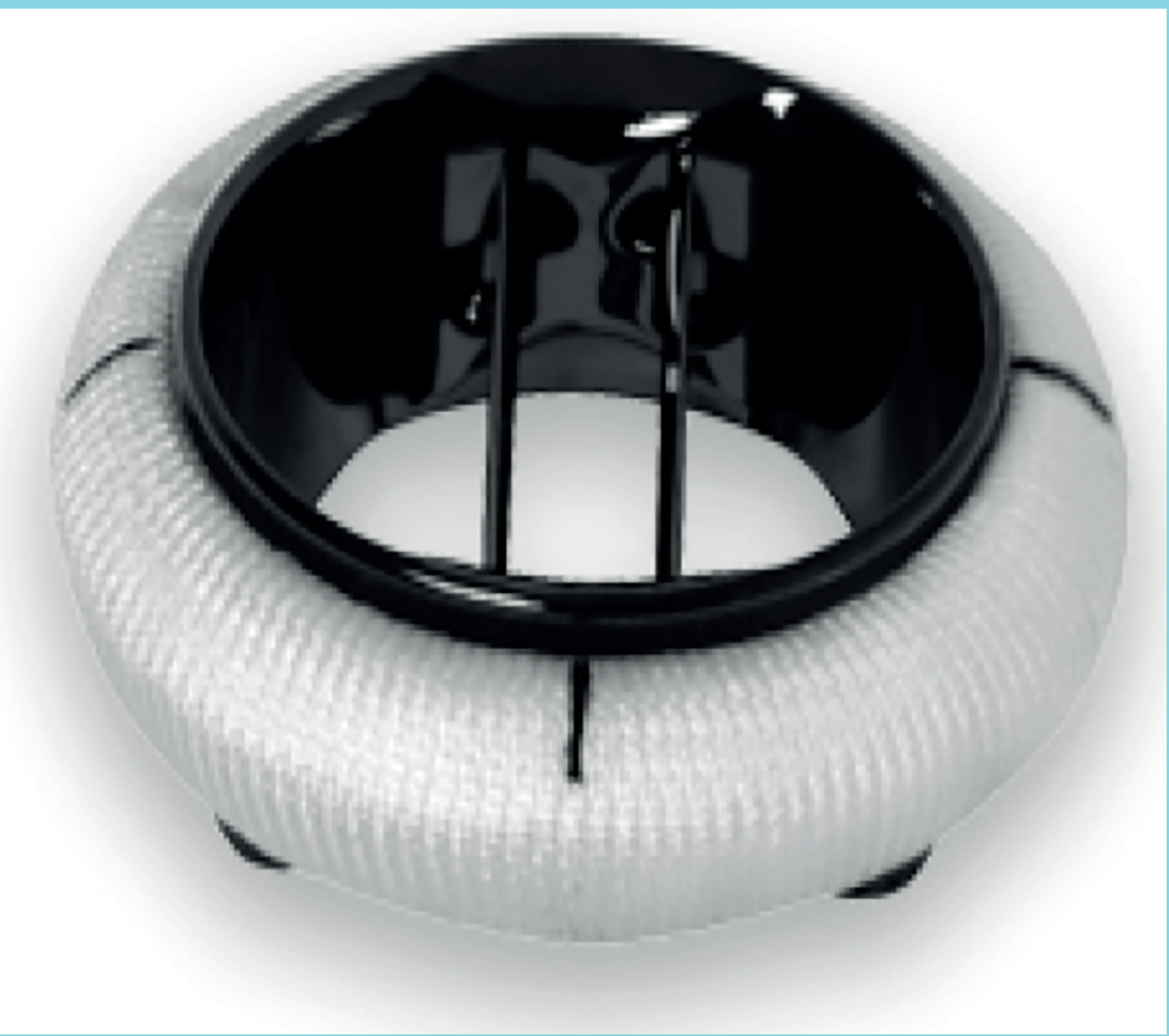




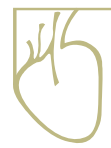

ta de una cavidad de orificio tubular y dos valvas de grafito recubiertos con carbono pirolítico, lo que proporciona una superficie más lisa, disminuyendo así la trombogenisidad. El anillo de sutura está construido con una tela de politetrafluoroetileno (PTFE). Esta prótesis OnX Conform viene en un único tamaño, 25/30, con un orificio efectivo de $2,1 \mathrm{~cm} 2$, manteniendo gradientes muy bajos, cercanos a 4,4 $\mathrm{mmHg}$. El diámetro interno del orificio es de $23,4 \mathrm{~mm}$ y el del anillo de
Estenosis mitral con calcificación severa del anillo mitral...

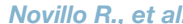

sutura de $39 \mathrm{~mm}$, con una altura de perfil abierta de $17,3 \mathrm{~mm}$. Esto hace que las valvas en sí se muevan dentro de un verdadero cilindro, disminuyendo al máximo las probabilidades de obstrucción al movimiento de las valvas por tejido residual infra protésico. A su vez, este extenso cojinete de sutura de PTFE se adapta a las irregularidades que pueda presentar el anillo mitral, disminuyendo en gran medida las probabilidades de desprendimientos peri protésicos.

\section{Referencias}

1. VEERAGANDHAM RS, GOLDIN MD. Surgical management of radiation-induced heart disease. Ann Thorac Surg 1998;65:1014-9.

2. DESAI MY, WU W, MASRI A, et al. Increased aorto-mitral curtain thickness independently predicts mortality in patients with radiation associated cardiac disease undergoing cardiac surgery. Ann Thorac Surg 2014;97:1348-55.

3. MILIND Y. DESAI, MD,A,* CHRISTINE L. JELLIS, MD, PHD,A,* RUPESH KOTECHA, MD,B DOUGLAS R. JOHNSTON, MD,ABRIAN P. GRIFFIN, MDA Radiation-Associated Cardiac Disease A Practical Approach to Diagnosis and Management. JACC Vol 11. NO 8. 2018

4. MILIND Y. DESAI, MD,A STEPHAN WINDECKER, MD, PHD,B PATRIZIO LANCELLOTTI, MD, PHD,c Prevention, Diagnosis, and Management of Radiation-Associated Cardiac Disease JACC Scientific Expert Panel. JACC Vol 74, NO 7. 2019

5. CASSELMAN FP, GILLINOV AM, MCDONALD ML, COSGROVE DM III. Use of the anterior mitral leaflet to reinforce the posterior mitral annulus after debridement of calcium. Ann Thorac Surg. 1999;68:261-2 